\title{
Opportunities to learn
}

\author{
Margaret Walshaw
}

Published online: 4 December 2012

(c) Springer Science+Business Media Dordrecht 2012

From a tradition that promoted procedural approaches to teaching, mathematics teacher education is now mindful of the social, affective, and cultural dimensions of teaching and learning. In case you missed it, mathematics teacher education over recent decades has steadily brought to the fore a wide range of notions including connections to everyday contexts, discursive interchanges, structured and purposeful tasks, as well as varied materials and representations. When these, and other, notions were conceived of as contributing to a productive learning community, it was only a matter of time before researchers would begin talking about opportunities to learn. Opportunities to learn, as you will be aware, can have a significant and sustained impact on student knowledge, attitude, self-esteem, independence, and confidence.

Opportunities to learn, of course, are wide-ranging within the pedagogical tool kit and include authentic applications that arise out of real-life contexts. The use of contexts has been shown to motivate student engagement, illustrate potential applications, provide a medium for mathematical reasoning and thinking, and anchor student understanding. Opportunities to learn also include scaffolding by the teacher through the use of selective tasks and visual representations. Purposeful tasks offer a vehicle for students to learn about particular mathematical ideas in ways that allow them to attend to the underlying mathematical structure. Multiple representations via stories, pictures, and symbols, on the other hand, lighten the cognitive load of the learner by providing conceptual tools for thinking. Let us not forget, too, that opportunities to learn also involve explicit instruction and purposeful classroom interchanges and feedback to students. A classroom environment in which the teacher orchestrates thoughtful discussion around meanings and understandings makes a contribution to the enhancement of student learning.

All these factors are associated with opportunities for students to learn. They lie at the heart of the articles in this issue. The first article investigates the views of teachers in relation to real-life connections made through story problems. In "Prospective elementary teachers' perceptions of real-life connections reflected in posing and evaluating story problems," Ji-Eun Lee explores what teachers know about story problems and their

M. Walshaw $(\bowtie)$

Massey University, Palmerston North, New Zealand

e-mail: m.a.walshaw@massey.ac.nz 
connections with mathematics and students' daily lives. Underpinning the exploration is a realization that story problems provide a link between mathematics and the real world. The participants in the study were 71 undergraduates enrolled in an elementary mathematics methods course. Through a series of tasks within the study, the participants were initially invited to provide a set of criteria for exemplary story problems that connected to real life. They also gathered as well as created exemplary story problems based on their criteria. From the participants' material, the researcher chose 10 different examples and then asked the participants to rate on a 5-point scale the closest connections between problem and real-life situations.

Findings revealed that around one-third of the story problems were sourced from printed texts, while two-thirds were found web-based resources. Money- and time-related contexts dominated and most focused on numbers, operations, and computational skills and required a numeric answer. Of note is the fact that of the criteria that the participants constructed, $42 \%$ were not directly related to real-life connectedness. For example, participants associated characteristics such as clear directions, age appropriateness, and challenge as significant criteria. Of significance for teacher education is the point that individual participants' assessments of the reality of the story problem contexts varied widely. The participants also differed in their understanding regarding what information within the story could be taken as assumed and what information required explicit explanation. If prospective teachers have neither a shared understanding of the reality within a story problem nor a shared understanding of the level of information necessary to solve it, opportunities to learn are significantly closed.

The second article in this issue also involves research with prospective teachers. JaneJane Lo was interested in what prior fractions knowledge a group of Taiwanese prospective elementary teachers brought with them to their beginning mathematics methods course. In "Prospective elementary teachers' knowledge of fraction division," she examined teachers' performance and strategies on two partition division-related problems. Employing the data collection tools of a one-to-one interview as well as a written questionnaire with 45 non-mathematics majors prospective teachers, Lo specifically investigated subject content knowledge and the ways in which the teachers represented fraction division through word problems or picture diagrams.

Lo found that her participants were proficient at solving fraction division problems. They also demonstrated flexibility in their reasoning by employing multiple solution strategies in solving fraction division word problems. They had a sound understanding of the concept of unitizing and of the part-whole relationship. However, less than half of the participants were able to pose a meaningful word problem that was correct for a given problem presented to them numerically. Lo raises the point that educators need to offer prospective teachers more opportunities to develop their word problem posing and more opportunities to learn in order to enhance their diagram proficiency for representing the solution of word problems.

The third article in the issue also picks up the point of opportunities to learn. The article takes us into the classroom to explore opportunities made available to students through classroom discussions. In "Mathematics discussion by design: Creating opportunities for purposeful participation," John Henning, Timothy McKeny, Gregory Foley and Megan Balong, investigated the nature and purposes of discussions that one seventh grade teacher facilitated and the relationship of those discussion with instructional design. Working from the premise that as the level of mathematical knowledge is enhanced through a unit of work, students' articulations of that knowledge are also enhanced, they asked: How does 
the instructional design for a series of mathematics discussions in a unit of work influence the interactions between the teacher and her students?

The lead researcher worked with the teacher in designing a series of nine class sessions for the twenty-one students of varying ability. In particular, they developed a unit on surface area and volume that would allow students to explore relationships among a number of shapes and to develop solution strategies for findings areas and volume. They developed a heuristic for designing discussions, and naming it 'the bow tie', they showed how it represented three unique discussion types, namely framing, conceptual, and application discussions. Using conversation analysis, the researchers found that framing discussions were shorter in duration and offered less teacher guidance and more encouragement. Conceptual discussions provided the highest evidence of teacher guidance and the lowest number of confirming responses. The highest level of student participation in discussion occurred during the application phase. The study offers insights for teachers in relation to understanding the different types of discussions and their individual purposes. Such understanding can contribute to enhancing student participation and the quality of mathematical discourse.

The authors of the three articles provide rich evidence that the opportunity for learning rests with planned and purposeful pedagogical practice. Selected story problems, a range of fraction representations, and structured classroom interaction all provide opportunities for students to learn. The effective use of instructional activities and communication, alongside resources and tools, is dependent on teachers listening and responding to what students say in a way that supports the development of significant mathematical thinking. Our authors reveal how that can happen. We hope that you enjoy their articles. 